\title{
LAS EMOCIONES: ¿INNECESARIAS PARA EL ENTENDIMIENTO CIENTÍFICO?
}

\author{
Arturo Ramos Argott*
}

El interés por caracterizar la noción del entendimiento científico es relativamente nuevo, no obstante que el término ha sido ampliamente utilizado en la literatura científica y filosófica, sin mencionar muchas otras. Como aportación a las investigaciones actuales sobre esta noción, el objetivo del siguiente escrito es presentar un análisis crítico de la relación entre el entendimiento científico y las emociones.

Palabras clave: entendimiento, entendimiento cientifico, actividad cognitiva, lógica, emociones.

THE EMOTIONS: UNNECESSARY FOR THE SCIENTIFIC UNDERSTANDING?

Scientific understanding has become a trending topic in the last few years notwithstanding that the term has been widely used in the philosophical and scientific literature-without mentioning other fields-for many years. As a contribution to the contemporary discussions about this theme, the goal of the following paper is to present a critical analysis of the relation between scientific understanding and emotions.

Keywords: understanding, scientific understanding, cognitive activity, logic, emotions.

* Universidad Nacional Autónoma de México-FES Acatlán, Santa Cruz Acatlán, México. Correo electrónico: arargott@apolo.acatlan.unam.mx 


\section{Introducción}

CON EL FIN DE DETERMINAR SI EXISTE O NO UNA RELACIÓN ENTRE LAS emociones y el entendimiento científico, observaremos si en el proceso de entender un fenómeno, dentro del contexto de la ciencia, las emociones juegan algún papel o no. Si encontramos que de hecho lo hacen, por supuesto habrá que averiguar cuál es ese papel ${ }^{1}$. Podemos formular la pregunta guía de la presente reflexión como sigue: ¿es acaso la emoción un ingrediente necesario para lograr el entendimiento científico de algún fenómeno?

La tesis que defenderé a lo largo de este escrito consta de dos puntos: por un lado mostraré que, de hecho, las emociones sí juegan un papel importante para el entendimiento, pero sólo para el entendimiento de tipo natural -que no es científico, aunque sí epistémico. Esto quiere decir que las emociones son una manera como el cuerpo entiende el mundo con el que se relaciona a través de la experiencia perceptual ${ }^{2}$.

Por otro lado, defenderé que las emociones no juegan ningún papel necesario para el entendimiento científico. En ciertos casos las emociones pueden acompañar el proceso del entendimiento científico pero no son necesarias. Esto quiere decir que no necesitamos de las emociones para entender científicamente el mundo, lo que necesitamos para hacerlo es: tener experiencias comunes y contar con algún tipo de lenguaje específico con el que podamos guiar intersubjetivamente ${ }^{3}$ las actividades cognitivas habilitadas por el entendi-

1 Cabe mencionar que la clase específica de fenómenos que pueden ser entendidos, dada la caracterización del entendimiento científico que desarrollaré en este escrito, es la clase de los fenómenos naturales que presentan un comportamiento constante y predictible. La repetición y la predicción son aquí la base del entendimiento científico. De esta manera, evitaré abordar el problema acerca de si debemos considerar que los fenómenos estocásticos, por tener naturaleza distinta de los fenómenos nomológicos, no pueden ser entendidos de la misma forma. Ejemplos de fenómenos estocásticos podemos encontrarlos en la física, algunos más en biología y quizás en la mayoría de los fenómenos del área de lo humano.

2 ¿Qué quiere decir que el entendimiento natural sea epistémico, pero no científico? Que el entendimiento natural involucra algún tipo de actividad cognitiva singularmente exitosa, esto es, exitosa para un solo individuo; y que por otro lado, el entendimiento científico involucra tipos de actividades cognitivas que necesariamente deben ser intersubjetivamente exitosas, esto es, igualmente exitosas para varios individuos.

3 La noción de objetividad se distingue de la de intersubjetividad en que la primera se enfoca más en la búsqueda llana de la verdad que en establecer los medios para que, en dado caso de que la verdad sea encontrada, esta pueda transmitirse a varios sujetos. En múltiples casos, el término intersubjetivo implica el de la objetividad dado que se puede apelar a este último para explicar la posibilidad de transmisión de conocimiento entre sujetos. 
miento científico ${ }^{4}$. Y que por tanto, el único papel que pueden desempeñar las emociones para el entendimiento científico es el de motivadores. Pero este papel no es necesario, dado que el entendimiento científico puede tener por causas otros motivadores que sólo las emociones; ejemplos de esto último son las necesidades biológicas y culturales, las evaluaciones teóricas y empíricas sobre nuestra situación epistémica, etcétera ${ }^{5}$.

Defenderé esta tesis tanto en el contexto de una teoría de la emociones fisiologista (James) $^{6}$, como en el contexto de una teoría cognitivista (Smith y Lazarus). La argumentación sigue más o menos la siguiente línea: (supuesto 1) si suponemos que para una teoría fisiologista las emociones son una respuesta a estímulos presentes del entorno, toda aquella actividad cognitiva, incluida la del entendimiento científico, que no involucre la percepción actual de estímulos presentes en el entorno ${ }^{8}$, carecerá de un correlato emocional'; por otro lado, y dentro de un contexto cognitivista, (supuesto 2) si suponemos que las emociones aparecen sólo después de que una situación ha sido cognitivamente evaluada, y si aceptamos que una situación puede evaluarse correctamente sólo cuando ha sido entendida exitosamente, entonces, aunque las emociones dependan del entendimiento para originarse correctamente, el entendimiento no depende de las emociones de ningún modo. Siendo este el caso, dado que el entendimiento científico permite realizar inferencias de manera abstracta y en un ambiente teorético virtual -lo que cancela la percepción actual de estímulos presentes en el entorno- (dado el supuesto 1) no será acompañado necesariamente por alguna emoción desde una postura fisiologista; y dado que una evaluación

4 Esto se percibirá mejor más adelante, cuando caracterice la noción del entendimiento científico.

5 Incluso si se argumentara que el deseo de entender algo puede derivarse en última instancia y de manera indirecta de una emoción, se tendría que mostrar una cadena deductiva de necesidad entre la existencia de la emoción y el deseo de entender algo. Supongamos que mi deseo de entender científicamente el fenómeno de la caída de los cuerpos (la gravedad), proviene de mi deseo previo de querer volar y evitar esta caída, y supongamos que mi deseo de volar y evitar la caída, proviene de mi deseo de salvar un abismo que me impide llegar rápidamente a mi destino, y supongamos, finalmente, que mi deseo de llegar rápidamente a mi destino proviene del miedo que me surge de llegar a mi destino lentamente, dado que entre más tiempo me tome más probable es que me asalten y me maten. En muchas cosas este tipo de argumento no convencería. ¿Cómo demostrar que la motivación emocional no se acabó en la primera parte de la cadena y las siguientes partes de la misma no dependen de ésta, sino de una reflexión puramente teórica que, aunque en un primer momento podía observarse que era acompañada por una emoción, en un momento posterior no?

6 JAMES, William, "What is an emotion?", Mind 9, (34), 1884, pp. 188-205.

7 Smith, Craig-Lazarus, Richard, "Emotion and Adaptation", en: Pervin, Lawrence (Ed.), Handbook of Personality: Theory and Research, Guilford, New York, 1990, pp. 609-637.

8 No analizaré la cuestión del acto de recordar y su relación con las emociones.

9 El entendimiento científico trabaja con conceptos en un lenguaje abstracto. Si yo escribo la palabra oso, el estímulo actual presente en el entorno que percibe en este momento el lector es la palabra oso, y no el oso en sí. Ahora, a menos que alguna vez la palabra oso haya salido de un texto y haya intentado comer al lector, oso no tendría por qué generar ninguna emoción, mucho menos el concepto al que el término oso pretende hacer referencia, otra vez, a menos que el concepto oso haya intentado salir del pensamiento del lector para comérselo, por lo que en el plano abstracto de los conceptos, se podrá generar entendimiento científico utilizando términos como oso, muerte, comer o correr, sin que ninguna emoción aparezca asociada a estas palabras o a sus conceptos. Para concluir, debe enfatizarse que entender y recordar son facultades cognitivas diferentes [Hume, 1896], que aunque pueden tener relación, individualmente implican actos del pensamiento distintos. Aceptado este punto, recordar al oso utilizando esta palabra, dado que el propósito del término, en este caso, no es referir al concepto sino al recuerdo, entonces aquí sí podría generar miedo, no la palabra en sí, sino el recuerdo asociado. 
cognitiva correcta de una situación depende de entender exitosamente la situación en cuestión, (dado el supuesto 2), entonces el entendimiento no depende de las emociones, aunque si la inversa en el contexto de una teoría cognitivista. Esto permite concluir que el entendimiento científico no tiene relación necesaria con las emociones en ninguna de las dos perspectivas abordadas sobre las emociones.

Para lograr todo lo anterior primero presentaré las caracterizaciones del entendimiento natural y científico sobre las que he trabajado durante varios años. Éstas, tienen su base en las teorías de Locke $^{10}$, Hume ${ }^{11}$, Peirce ${ }^{12}$ y Dewey ${ }^{13}$. Una vez hecho lo anterior me encargaré de exponer y analizar la teoría de las emociones en sus vertientes fisiologistas y cognitivistas para mostrar después que, si se acepta la caracterización del entendimiento científico que doy en este escrito, entonces las emociones no tienen ninguna relación necesaria con éste.

\section{El entendimiento natural}

El entendimiento es el conjunto de las operaciones cognitivas -inferencias, relaciones, comparaciones, etc.- que realizamos sobre fenómenos, situaciones y entidades del mundo, de manera reactiva y no-reflexiva, ante estímulos específicos del entorno y con fines prácticos, biológicos, o de otra índole.

Los fundamentos de esta caracterización del entendimiento se encuentran en autores como Locke y Hume. Estos filósofos fueron dos de los primeros en sugerir la existencia de un entendimiento natural. Para estos autore,s el entendimiento es una facultad cognitiva con la que nacemos que nos permite realizar "operaciones mentales"14 y cuya ejecución repetida, debido al enfrentamiento constante con una misma situación, produce que se conviertan en un "hábito" (Hume) $)^{15}$. Acerca de las operaciones mentales facultadas por nuestro entendimiento, Locke sostiene al inicio del Libro Segundo de su Ensayo:

En segundo lugar, la otra fuente de donde la experiencia proporciona ideas al entendimiento es la percepción de las operaciones que realiza nuestra mente interiormente, según es aplicada a las ideas que ha obtenido. Tales operaciones, cuando el alma llega a

10 Locke, John, Ensayo sobre el Entendimiento Humano, Gernika, México, 1998.

11 Hume, David, A Treatise on Human Nature, Selby-Bigge, L.A. (Ed.), Clarendon Press, Oxford, 1896. Hume, D., An Enquiry concerning Human Understanding, BENNETT, Jonathan (Ed.), 2008, disponible en: http://www. earlymoderntexts.com/pdfs/hume1748.pdf.

12 Peirce, C.S., "Principles of Philosophy", en: Hartshorne, Charles-Weiss, Paul (Ed.), Collected papers of Charles Sanders Peirce, Vol. I, Harvard University Press, Cambridge, 1931. Peirce, C.S., "Pragmatism and Pragmaticism", en: Hartshorne, Charles-Weiss, Paul (Ed.), Collected papers of Charles Sanders Peirce, Vol. V, Harvard University Press, Cambridge, 1934.

13 Dewey, J., Logic. The Theory of Inquiry, Henry Holt and Company, New York, 1938.

14 Locke, J., Ensayo sobre el Entendimiento Humano, op. cit., p. 16.

15 Cfr. Hume, D., A Treatise on Human Nature, op. cit. 
considerarlas y a reflexionar sobre ellas, suministran al entendimiento otro conjunto de ideas que no pueden alcanzarse de las cosas externas ${ }^{16}$.

Según Locke, es por medio de ese percibir nuestro mundo interno -la reflexión- que podemos captar las operaciones que realiza nuestra mente sobre los contenidos objetos del pensamiento. Para este autor, el mobiliario del mundo interno de nuestras mentes se compone de dos clases: la clase de los objetos sobre los que piensa el pensamiento, y la clase de las actividades del pensamiento con las que se piensa sobre los objetos del pensamiento. En su teoría, las ideas, provenientes de los sentidos externos, o del "sentido interno"17, son elementos pertenecientes a la primera clase, y las operaciones del entendimiento, elementos pertenecientes a la segunda clase.

¿Qué tipo de operaciones son éstas que puede realizar nuestro entendimiento? Locke ya no es muy claro sobre este punto, y dice: "aquí utilizo el término operaciones en un sentido amplio, de modo que comprende no solamente las acciones de la mente acerca de sus ideas, sino también cierta clase de pasiones que en ocasiones surge de ellas, como la insatisfacción o incomodidad que se deriva de algún pensamiento"18. A este respecto es Hume quien desarrolla una mejor explicación del tipo de operaciones que se producen gracias a nuestro entendimiento. Para este autor, las diferentes relaciones que podemos establecer entre los contenidos objetos de nuestro pensamiento - de cantidad, de calidad, de igualdad, etc. - son el resultado de las operaciones cognitivas de nuestro entendimiento ${ }^{19}$.

La investigación naturalista sobre el entendimiento es una de las mayores contribuciones hechas por Hume a la filosofía ${ }^{20}$. Para este autor es un hecho observable que la mente de los animales, incluidos los humanos, opera estableciendo relaciones de varios tipos y de maneras sistemáticas: "the mind's thoughts or ideas are obviously inter-connected in some systematic way: there is some order and regularity in how, in memory and imagination, one idea leads on to another" ${ }^{21}$. Para Hume, de las diferentes relaciones entre los contenidos objeto que el pensamiento puede establecer, la más importante en términos biológicos es la de la "causalidad"22. Su importancia radica tanto en que la encontramos a la base de todas nuestras predicciones, como en que, por medio de ella, establecemos asociaciones entre estímulos, ideas y acciones. Hume lo dice así:

16 Locke, J., op. cit., p. 141.

17 Idem.

18 Ibidem, p. 142.

19 Hume, D., A Treatise on Human Nature, op. cit., p. 12. El predicado "cognitivo" asociado al término actividad para hacer referencia al entendimiento no es propio de Hume ni de Locke, sino producto de mi investigación.

20 De hecho, el estudio que efectuó sobre las operaciones del entendimiento es uno secundario pero fundamental, su interés principal siempre fue, desde el principio, la explicación de la posibilidad del conocimiento desde una perspectiva naturalista, psicologista y pragmatista. No obstante, para lograr su objetivo, Hume tuvo que estudiar la naturaleza de nuestras facultades cognitivas.

21 Hume, D., An Enquiry concerning Human Understanding, op. cit., p. 10.

22 Hume, D., A Treatise on Human Nature, op. cit., p. 45. 
The idea of sinking is so closely connected with that of water, and the idea of suffocating with that of sinking, that the mind makes the transition without the assistance of the memory. The custom operates before we have time for reflexion. The objects seem so inseparable, that we interpose not a moment's delay in passing from the one to the other. But as this transition proceeds from experience, and not from any primary connexion betwixt the ideas, we must necessarily acknowledge, that experience may produce a belief and a judgment of causes and effects by a secret operation, and without being once thought of... For we here find, that the understanding or imagination can draw inferences from past experience, without reflecting on it; much more without forming any principle concerning $i$, or reasoning upon that principle ${ }^{23}$.

Según Hume, la asociación causal es una de las herramientas cognitivas que mejor nos permite sobrevivir en este mundo. Gracias a ésta podemos predecir y, por ende, fomentar o evitar eventos de la naturaleza. En el ejemplo humeano de la cita anterior, gracias a que podemos anticipar que la causa del ahogamiento es el hundimiento en el agua, es que podemos realizar la acción de detenernos ante un río. Para este autor, las asociaciones causales conformadas por el entendimiento natural se generan automáticamente debido a la repetición, y, una vez establecidas, se ejecutan mecánicamente gracias al hábito. En su visión, el cuerpo es un autómata con la facultad natural de asociar estímulos, ideas y acciones por medio de la repetición. Siendo esto así, en el entendimiento natural no hay reflexión ni deliberación, por lo que, cuando entendemos naturalmente el mundo, no somos diferentes de los animales, ni ellos de nosotros.

Las ventajas que presenta el entendimiento natural, caracterizado así, frente a otros tipos de entendimiento, como por ejemplo el científico, son: (i) que realiza las asociaciones entre ideas y acciones de manera muy rápida y (ii) que requiere de poca energía. Estas ventajas son el resultado de varias investigaciones descritas y analizadas por Hassin, Bargh, y Uleman ${ }^{24}$ :

Inferring causal relations among the constituent elements of this world, be they physical objects or living things, is one of the most important tasks the cognitive system has to perform... Moreover, in a dynamic world, full of shifting conditions and sudden surprises, inferring causes is a task that the cognitive system has to perform very frequently and very swiftly. This frequent need for rapid causal understanding might place an overwhelming demand for cognitive resources. Since these resources are limited [Kahneman $\left.{ }^{25}\right]$, it seems that some of the work related to inferring causality has to be done in a way that demands less cognitive resources, that is-automatically ${ }^{26}$.

23 Ibidem, p. 60.

24 Hassin, Ran, Bargh, John, Uleman, James, "Spontaneous causal inferences", Journal of Experimental Social Psychology, (38), 2002, pp. 515-522.

25 Kahneman, Daniel, Attention and Effort, Prentice-Hall, New Jersey, 1973.

26 Ibidem, p. 515. 
La rapidez de las inferencias del entendimiento natural se debe a que se conforman hábitos de inferencia; hábitos que se efectúan de manera mecánica y se establecen de manera automática. Para adquirir un hábito de inferencia es necesario que las experiencias perceptivas se hayan repetido un número considerable de veces, generando, así, una asociación estímulo-idea-acción. Esto quiere decir que las asociaciones entre estímulos, ideas y acciones en el plano del entendimiento natural no se efectúan a priori ni de manera deliberada ${ }^{27}$. Para ejecutarse, esta facultad cognitiva en su faceta natural depende totalmente de la experiencia perceptual presente, es decir, del estímulo y la repetición. Así, con el entendimiento natural nunca podríamos inferir un hecho consecuente sin que el hecho antecedente -el estímulo- haya sido provisto por la percepción en un momento actual.

¿Qué papel juegan las emociones para esta facultad natural? De acuerdo con las ideas de James ${ }^{28}$, uno de los autores clásicos de la teoría fisiologista de las emociones, las emociones son el enlace entre el estímulo y la acción. Son éstas las que vuelven mecánico y económico el proceso de asociar un estímulo del entorno con una acción:

Now among these nervous anticipations are of course to be reckoned the emotions, so far as these may be called forth directly by the perception of certain facts. In advance of all experience of elephants no child can but be frightened if he suddenly find one trumpeting and charging upon him. No woman can see a handsome little naked baby without delight, no man in the wilderness see a human form in the distance without excitement and curiosity. I said I should consider these emotions only so far as they have bodily movements of some sort for their accompaniments ${ }^{29}$.

En suma, desde la perspectiva de estos autores, es posible capturar la idea de que el entendimiento implica la efectuación de procesos cognitivos precisos que brindan resultados epistémicos diversos, siendo uno de ellos la evaluación correcta de las situaciones que se perciben. Además, dado que el entendimiento es natural mientras no se efectúe deliberadamente, entonces, siendo natural, implica la ejecución mecánica no-reflexiva de las operaciones cognitivas de nuestro entendimiento, que se realizan con fines prácticos, sean biológicos de supervivencia y reproducción, sean de otra índole. Su principal virtud es la rapidez con que se realizan, principalmente debido a las emociones que las activan y las guían, pero con la desventaja de que sólo se ejecutan ante el entorno o situación pertinente. En conclusión, con el entendimiento natural sólo entendemos los fenómenos cuando se presentan los estímulos específicos, y en razón de esto es que podemos afirmar que las operaciones producto de este entendimiento son acompañadas, e incluso posibilitadas, por ciertas clases de emociones, lo cual no sucede con el entendimiento científico.

27 Hume, D., A Treatise on Human Nature, op. cit., p. 60.

28 James, W., "What is an emotion?", op. cit.

29 Ibidem, p. 191 


\section{El entendimiento científico}

El entendimiento científico es el conjunto de las operaciones cognitivas -inferencias, relaciones, asociaciones, etc.- que realizamos deliberadamente y de manera ordenada para conseguir alguna o algunas de las metas epistémicas clásicas de la ciencia -descripción, explicación y predicción- sobre clases de fenómenos, situaciones y entidades de la naturaleza. Los puntos a resaltar en esta sección son: (a) que las operaciones cognitivas del entendimiento se realizan deliberadamente en un contexto científico; y (b) que los resultados que obtenemos por medio del entendimiento científico implican, en un caso, la evaluación exitosa de las situaciones. Acerca de (a), si las operaciones cognitivas se realizan deliberadamente, entonces no se realizan de manera reactiva y, como veremos más adelante, dado que las emociones en una postura fisiologista surgen sólo como reacción a estímulos específicos del entorno, entonces entendimiento y emociones son dos cosas diferentes sin relación. Acerca de (b), dado que en una teoría cognitivista de las emociones, éstas sólo surgen después de realizar una evaluación cognitiva correcta de la situación, y si una evaluación cognitiva correcta de una situación es consecuencia de entender exitosamente esa situación, entonces el entendimiento no depende de las emociones, aunque sí la inversa ${ }^{30}$.

Los fundamentos teóricos de la caracterización del entendimiento científico con la que inicié esta sección se encuentran en las teorías de Peirce y Dewey ${ }^{31}$. Para Peirce, la lógica es, por un lado, el estudio de las operaciones cognitivas que realizamos de manera deliberada; cuando la psicología, por el otro, es el estudio de las operaciones cognitivas que son realizadas por nuestra mente de manera mecánica y reactiva. Para lograr deliberar sobre nuestras operaciones cognitivas, es necesario primero tener auto-control sobre ellas. Peirce lo dice así: "Logic is the theory of self-controlled, or deliberate, thought" 32 . Más adelante, agrega: "consequently, to say that an operation of the mind is controlled is to say that it is, in a special sense, a conscious operation; and this no doubt is the consciousness of reasoning" 33 . Desde su perspectiva, las operaciones cognitivas que hacemos con nuestro entendimiento pueden caer bajo nuestro control; y cuando esto sucede, es que podemos realizar estudios lógicos sobre las propiedades que las mismas poseen.

Por otro lado, desde la teoría de la lógica del filósofo pragmatista John Dewey ${ }^{34}$, es posible captar que, entre las operaciones cognitivas que se realizan naturalmente y aquellas que se realizan deliberadamente, existe una distinción solamente cualitativa. Esto quiere

30 En este texto asumiremos que la evaluación correcta de una situación depende de entenderla previamente y de manera exitosa.

31 Ya tengo alrededor de cuatro años revisando esta caracterización.

32 PeIRCE, C.S., "Principles of Philosophy", op. cit., $\$ 1.191$.

33 PeIrCE, C.S., "Pragmatism and Pragmaticism", op cit., §5.441.

34 Cfr. Dewey, J., Logic. The Theory of Inquiry, op. cit. 
decir que las operaciones que podemos hacer en el plano de la ciencia son las mismas que se realizan en el plano natural, con la diferencia de que en el plano de la ciencia éstas se realizan de manera deliberada. Dewey lo dice así:

At the outset, the habit that operates in an inference is purely biological. It operates without our being aware of it. We are aware at most of particular acts and particular consequences. Later, we are aware not only of "what is done from time to time but of how it is done. Attention to the way of doing is, moreover, indispensable to control of what is done. The craftsman, for example, learns that if he operates in a certain way the result will take care of itself, certain materials being given. In like fashion, we discover that if we draw our inferences in a certain way, we shall, other things being equal, get dependable conclusions $^{35}$.

Como lo establece Dewey, cuando logramos estar conscientes de la existencia de estas operaciones cognitivas que somos capaces de hacer por naturaleza, podemos eventualmente ser conscientes de lo que conseguimos con estas operaciones y de cómo lo conseguimos. Si logramos darnos cuenta de lo anterior -según Dewey-, podemos entonces llegar a controlarlas. Y en los casos en los que las controlemos, serán los casos en donde necesariamente dejarán de efectuarse de manera mecánica y reactiva por causa del entorno.

En suma, desde la perspectiva conjunta de estos dos autores es posible concebir al entendimiento científico como el conjunto de las operaciones cognitivas que realizamos deliberadamente -de manera no-reactiva- para conseguir distintas metas, en especial del tipo epistémicas.

\section{Las emociones}

En esta sección no intentaré resolver ni abordar todos los problemas complejos que existen entre las visiones opuestas de las teorías fisiologistas y cognitivistas sobre las emociones. Mi objetivo principal, tal como lo establecí en la introducción, es mostrar que las emociones, en cualquiera de estas visiones, y así como son descritas por sus defensores, no juegan ningún papel necesario para las actividades cognitivas deliberadas del entendimiento científico, de acuerdo a como lo he caracterizado. Señalaré, entonces, sólo algunas de las características de estas teorías, que debido especialmente a la fuerza con la que las defienden, permiten conseguir el objetivo trazado desde el inicio.

\section{a) La emoción en una teoría fisiologista}

Para poder defender en el contexto de una teoría fisiologista que las emociones no

35 Dewey, J., Idem., p. 12. 
tienen relación necesaria con el entendimiento, se requiere satisfacer dos condiciones: (1) mostrar que no siempre estamos emocionados -si siempre estuviéramos emocionados, entonces todo lo que hiciéramos se encontraría relacionado con la emoción, incluidas las operaciones cognitivas del entendimiento; y (2) mostrar que toda emoción surge sólo por reacción a estímulos precisos del entorno. Si se cumplen estas dos condiciones, si no siempre estamos emocionados, y si toda emoción surge sólo como reacción al entorno, y si se acepta que el entendimiento científico, por ser deliberado, puede realizarse incluso cuando no hay percepción alguna del entorno ${ }^{36}$, entonces el entendimiento científico no tiene relación necesaria con las emociones, porque tanto puede ser activado por otra causa que no sea una emoción, como porque puede efectuarse sobre un objeto no percibido en el momento de su entendimiento.

\section{a.1) Relación fuerte entre el entorno y las emociones}

En las teorías fisiologistas sobre las emociones podemos capturar la idea de que uno de los propósitos fundamentales de sus argumentos es defender una relación fuerte entre el entorno y el sujeto, resistiendo así las tesis de que este último es una entidad emocionalmente abstracta desligada del mundo, que delibera y actúa separándose del curso natural de la realidad. Visiones como esta última llevan eventualmente a plantear una realidad emocionalmente intelectual de naturaleza distinta que la del cuerpo, idea a la que se oponen los fisiologistas. Para oponerse a esto es que autores como James ${ }^{37}$ terminan relacionando fuertemente las emociones con las experiencias perceptuales.

La idea de que las emociones y el entorno se encuentran fuertemente relacionadas la observamos en la siguiente afirmación de James: "to begin with, readers of this Journal do not need to be reminded that the nervous system of every living thing is but a bundle of predispositions to react in particular ways upon the contact of particular features of the environment" ${ }^{\prime 38}$. De acuerdo con esta visión, las emociones son producto de la interacción corporal con el ambiente material. No es necesaria una evaluación cognitiva de la situación para generar una reacción emotiva. De esta forma se establece una especie de "cerradura" 39 que sólo es abierta por el estímulo específico del entorno.

\section{a.2) No siempre estamos emocionados}

No obstante la relación fuerte que establece entre las emociones y el entorno, para el mismo James no siempre nos encontramos emocionados, es decir, no siempre estamos

\footnotetext{
36 Ejemplo: podemos entender el fenómeno de la caída de los objetos hacia la tierra por medio de las leyes físicas, incluso cuando no estamos viendo ningún objeto caer en un momento dado.

37 Cfr. JAMES, W., "What is an emotion?", op. cit.

38 Ibidem, p. 190.

39 Ibidem, p. 191.
} 
percibiendo el mundo externo y, en ese sentido, recibiendo estímulos. Esto nos indica que, en ciertos momentos, podemos realizar o manifestar otro tipo de actividades que no tienen relación con la emoción. Sobre estos estados neutrales sin emoción alguna, James sostiene:

If we fancy some strong emotion, and' then try to abstract from our consciousness of it all the feelings of its characteristic bodily symptoms, we find we have nothing left behind, no "mind-stuff" out of which the emotion can be constituted, and that a cold and neutral state of intellectual perception is all that remains ${ }^{40}$.

Más aún, James dice que, para estudiar a las emociones, es necesario detener el flujo intenso de la emoción en cuestión y llegar a un estado más tranquilo desde el cual poder abstraer de ella sus propiedades constitutivas:

The next thing to be noticed is this, that every one of the bodily changes, whatsoever it be, is felt, cutely or obscurely, the moment it occurs. If the reader has never paid attention to this matter, he will be both interested and astonished to learn how many different local bodily feelings he can detect in himself as characteristic of his various emotional moods. It would be perhaps too much to expect him to arrest the tide of any strong gust of passion for the sake of any such curious analysis as this; but he can observe more tranquil states, and that may be assumed here to be true of the greater which is shown to be true of the less ${ }^{41}$.

Es así que, si concedemos que las emociones están fuertemente ligadas al entorno y que no siempre nos encontramos en estados perceptivos ligados con emociones, entonces, dada la caracterización previa del entendimiento científico, las emociones, de acuerdo con una teoría fisiologista, no juegan ningún papel necesario para éste. El entendimiento científico no depende de los estímulos perceptuales actuales provenientes del entorno, ni para activarse, ni para realizarse: puedo inferir que si algo es fuego, entonces quema en un plano puramente teórico, es decir, puedo hacer la inferencia mencionada incluso cuando no hay nada en el entorno que sea fuego o que tenga que ver con el fuego. Y debido a que no hay emoción sin estímulo perceptual, entonces no hay emociones en las inferencias del entendimiento científico, porque este último no necesita de la experiencia perceptual presente. En definitiva, el único papel que pueden jugar las emociones es el de motivadores, pero no realizan este papel de manera exclusiva.

\section{b) La emoción en una teoría cognitivista}

Las teorías cognitivistas sobre las emociones se enfocan en defender principalmente la postura de que los procesos intencionales cognitivos que se encargan de evaluar las re- 
laciones sujeto-entorno son las causantes de las respuestas viscerales que caracterizan la emoción en términos fisiológicos ${ }^{42}$. En estas visiones entonces, el cuerpo no se encuentra ligado al entorno de manera directa, sino a través de procesos cognitivos evaluativos. Si esto es cierto, entonces los procesos cognitivos determinan de manera intelectual sobre qué y con qué intensidad debemos sentirnos emocionados en función de los fines, objetivos e ideales perseguidos por el sujeto. En esta perspectiva, si el entendimiento es la ejecución de operaciones cognitivas que nos brindan resultados, como por ejemplo, evaluaciones correctas sobre si debemos sentirnos emocionados o no, entonces entender una situación es condición previa para poder sentirla emocionalmente. Por ejemplo, entender que la muerte de un familiar es un factor fundamental para sentir tristeza por la falta del pariente en cuestión, es condición necesaria previa para poder -entonces- sentir la tristeza por la falta de esa persona. Dado esto, entonces, desde una teoría cognitivista las emociones no son necesarias para el entendimiento, aunque el entendimiento sí lo sea para las emociones.

Para mostrar que en las teorías cognitivistas el papel de la evaluación cognitiva es fundamental para la generación de una emoción presentaré las ideas de Smith y Lazarus ${ }^{43}$, dos de los más importantes defensores de la teoría valorativa (appraisal theory) de las emociones. Dichos autores sostienen:

Unlike physiological needs, which are internal and reliably signaled by specific stimulus conditions, adaptationally significant external events present themselves to complex species in a variety of guises. For instance, one class of events with which the organism must be prepared to cope in order to survive is that of threats to its well-being. All threats share the property of having the potential of resulting in harm if they are not avoided or neutralized ${ }^{44}$.

Para aquéllos, las emociones no pueden ser puramente fisiológicas, porque de ser este el caso, no podríamos explicar el proceso de cambio de las relaciones estímulos-emociones durante el periodo de adaptación de los organismos a situaciones nuevas. Por esto, para los autores de esta postura cognitivista, se vuelve necesario postular la existencia de procesos cognitivos evaluativos que incorporen la información pertinente de las nuevas situaciones a los esquemas cognitivos de conducta, incluidas las emociones. Así, dado que para los autores de estas posturas el fenómeno complejo de la adaptación de los organismos a nuevas situaciones no es algo determinado de antemano, las evaluaciones cognitivas se vuelven necesarias para explicar tal fenómeno. De acuerdo con ellos, esta tarea de adaptación sobrepasa las capacidades instintivas limitadas del cuerpo, y es por esto que la función cognitiva se vuelve necesaria:

\footnotetext{
42 Cfr. Sмith, Craig-Lazarus, Richard, "Emotion and Adaptation", op. cit. 43 Ibidem.

44 Ibidem, p. 614.
} 
However, because there is no simple mapping between objective stimulus properties and adaptive significance, the task of detecting significant events becomes quite formidable, and to accomplish it the organism must be able to somehow classify what is being confronted into a relatively small number of categories, corresponding to the various kinds of harm or benefit it may face. Above all, the emotional response is not a reaction to a stimulus, but to an organism (person)-environment relationship ${ }^{45}$.

En suma, si es cierto que antes de generar una asociación estímulo-emoción exitosa para un nuevo entorno, es necesaria la tarea de evaluar tal nueva situación para encontrar la conducta más adecuada con respecto a ella, y si el entendimiento precisamente implica la realización de las operaciones cognitivas con las que se evalúa la información del entorno novedoso, entonces el entendimiento no necesita de las emociones, ni como motivadores, ni como parte del proceso de evaluación inteligente de la situación, por el contrario, son las emociones las que dependerían de entender antes la situación.*

\section{Bibliografía}

Dewey, John, Logic. The Theory of Inquiry, Henry Holt and Company, New York, 1938.

Hassin, Ran, Bargh, John, Uleman, James, "Spontaneous causal inferences", Journal of Experimental Social Psychology, (38), 2002, pp. 515-522.

Hume, David, A Treatise on Human Nature, Selby-Bigge, L.A. (Ed.), Clarendon Press, Oxford, 1896.

, An Enquiry concerning Human Understanding, Bennett, JonAthan (Ed.), 2008, disponible en: http://www.earlymoderntexts.com/pdfs/hume1748.pdf

James, William, “What is an emotion?”, Mind 9, (34), 1884, pp. 188-205.

Kahneman, Daniel, Attention and Effort, Prentice-Hall, New Jersey, 1973.

Locke, John, Ensayo sobre el Entendimiento Humano, Gernika, México, 1998.

Peirce, C.S., "Principles of Philosophy", en: Hartshorne, Charles-Weiss, Paul (Eds.), Collected papers of Charles Sanders Peirce, Vol. I, Harvard University Press, Cambridge, 1931.

45 Idem.

* Artículo recibido: 29 de noviembre de 2014. Aceptado: 16 de diciembre de 2014. 
-------------, "Pragmatism and Pragmaticism”, en: Hartshorne, Charles-Weiss, Paul (Eds.), Collected papers of Charles Sanders Peirce, Vol. V, Harvard University Press, Cambridge, 1934.

Smith, Craig-Lazarus, Richard, "Emotion and Adaptation", en: Pervin, Lawrence (Ed.), Handbook of Personality: Theory and Research, Guilford, New York, 1990, pp. 609-637. 